\title{
Mannitol Dosing Error during Pre-neurosurgical Care of Head Injury: A Neurosurgical In-Hospital Survey from Ibadan, Nigeria
}

\author{
Amos Olufemi Adeleye ${ }^{1,2}$ Toyin Ayofe Oyemolade 2 \\ Oghenekevwe Efe Okere ${ }^{2}$
${ }^{1}$ Department of Surgery, Division of Neurological Surgery, College of Medicine, University of Ibadan, Ibadan, Nigeria
${ }^{2}$ Department of Neurological Surgery, University College Hospital, Ibadan, Nigeria

Toluyemi Adefolarin Malomo²

\begin{abstract}
Address for correspondence Amos Olufemi Adeleye, MBBS, Department of Neurological Surgery, University College Hospital, UCH, Ibadan, Owo, PMB 1053, Nigeria

(e-mail: femdoy@yahoo.com).
\end{abstract}

J Neurosci Rural Pract:2021;12:171-176

\begin{abstract}
Keywords

- mannitol

- inappropriate use

- dosing error

- head injury

- brain injury

- pre-neurosurgery
\end{abstract}

Objectives Inappropriate use of mannitol is a medical error seen frequently in pre-neurosurgical head injury ( $\mathrm{HI}$ ) care that may result in serious adverse effects. This study explored this medical error amongst $\mathrm{HI}$ patients in a Nigerian neurosurgery unit. Methods We performed a cross-sectional analysis of a prospective cohort of $\mathrm{HI}$ patients who were administered mannitol by their initial non-neurosurgical health care givers before referral to our center over a 22-month period.

Statistical Analysis A statistical software was used for the analysis with which an $\alpha$ value of $<0.05$ was deemed clinically significant.

Results Seventy-one patients were recruited: 17 (23.9\%) from private hospitals, $13(18.3 \%)$ from primary health facilities (PHFs), 20 (28.2\%) from secondary health facilities (SHFs), and 21 (29.6\%) from tertiary health facilities (THFs). Thirteen patients (18.3\%) had mild HI; 29 (40.8\%) each had moderate and severe HI, respectively. Pupillary abnormalities were documented in five patients $(7.04 \%)$ with severe $\mathrm{HI}$ and neurological deterioration in two with mild HI.

Mannitol administration was deemed appropriate in only 43.7\% (31/71). Data on mannitol dosing in $60.6 \%(43 / 71)$ of the patients showed $8 / 43(18.6 \%)$ receiving continuous $10 \%$ mannitol infusion. The remaining $35 / 43$ received mannitol as a $20 \%$ solution but also showing dosing error in $62.9 \%$ (22/35): overdosing in $7 / 35$ (20\%), and nonbolus administration in 15/35 (42.9\%). The distribution of the dosing error among the referring health facilities (all the 13 [100\%] patients from private hospitals, 66.7\% from PHF, $60 \%$ from SHF, and $45.5 \%$ from THF) showed a trend of better performance $(p=0.002)$ by the THFs.

Conclusion Mannitol use is apparently fraught with an understudied medical error in the pre-neurosurgical care of the head injured.
DOI https://doi.org/ 10.1055/s-0040-1722093 ISSN 0976-3147.

\footnotetext{
(c) 2021. Association for Helping Neurosurgical Sick People.

This is an open access article published by Thieme under the terms of the Creative Commons Attribution-NonDerivative-NonCommercial-License, permitting copying and reproduction so long as the original work is given appropriate credit. Contents may not be used for commercial purposes, or adapted, remixed, transformed or built upon. (https://creativecommons.org/licenses/by-nc-nd/4.0/)

Thieme Medical and Scientific Publishers Pvt. Ltd., A-12, 2nd Floor, Sector 2, Noida-201301 UP, India
} 


\section{Introduction}

Head injury (HI) remains a significant cause of morbidity, mortality, and great economic loss globally. ${ }^{1,2}$ In Nigeria, like most developing countries, a significant proportion of head injured patients are first seen at facilities without neurosurgical services. ${ }^{3,4}$ In these settings, there exists no standardized protocol for the care of these patients; therefore, diverse drug and nondrug medical agents are routinely administered. ${ }^{3}$ Mannitol is widely used to control intracranial hypertension following HI. ${ }^{5}$ In this regard, it is many times used as a temporizing measure between the time of the clinical recognition of intracranial traumatic emergencies and definitive operative care, this, sometimes in patients requiring transfers from the pre-neurosurgical health facilities (HFs) to specialist centers. ${ }^{5-7}$

Although the potency of mannitol to control acutely elevated intracranial pressure (ICP) in many neurological conditions, especially brain trauma, is well-known, little evidence exists to support its prophylactic use for this indication. ${ }^{7}$ Moreover, it is not all that a totally innocuous drug. Its use may actually result in various serious adverse effects including hypotension, electrolyte derangements, nephrotoxicity, and even rebound worsening of cerebral edema ${ }^{8,9}$ hence, the need for its rational use.

In the setting of $\mathrm{HI}$ in particular, the Brain Trauma Foundation (BTF) guidelines recommend that the use of mannitol prior to ICP monitoring be tailored only to patients with progressive neurological deterioration that is attributable to intracranial brain shifts/herniations, and at a dose of 0.25 to $1 \mathrm{~g} / \mathrm{kg}$ body weight. ${ }^{6,10}$

Our university teaching hospital is the nation's foremost center of neurosciences, receiving referrals for neurosurgical care of $\mathrm{HI}$ of all severities from most of the country. ${ }^{4,11}$ The details of the referral notes in many cases show not only wrong doses of mannitol but even inappropriate/unnecessary administration of this drug in many cases. And in some others, patients arrive our hospital following several hours of interstate referral to us with mannitol infusions, $20 \%$ or even $10 \%$ solutions, running ever so slowly.

In this study, the aim is to do a data-driven presentation of the presence and scope of this medical error in a prospective cohort of cases of $\mathrm{HI}$ referred to us for neurosurgical care in Ibadan, Nigeria.

\section{Methods}

This was a prospective cross-sectional study using our in-hospital registry of $\mathrm{HI}$ patients seen by us over a 22-month period ending in November 2017. All head injured patients with referral letters stating mannitol administration were recruited into this survey ( $\boldsymbol{- \text { Fig. }} \mathbf{1}$ ). The clinical information analyzed included the age and gender of the patients, severity of the HI using the Glasgow coma scale (GCS), the referring hospital type (whether primary/secondary/tertiary or private HFs), timing of mannitol administration with respect to neuroimaging, the formulations of the administered mannitol (i.e., 10 or $20 \%$ solutions) and the need or otherwise for the mannitol administration. The primary health

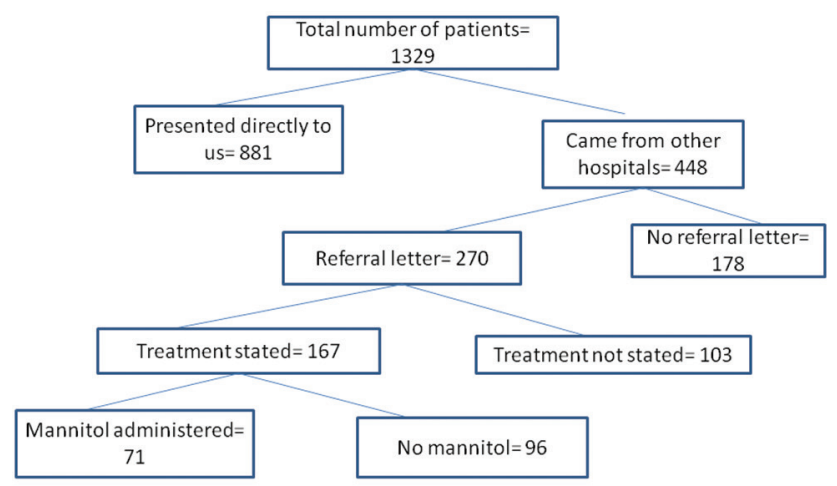

Fig. 1 Mannitol dosing error during pre-neurosurgical care of the head injured: flowchart showing how we arrived at the study population.

facilities (PHFs), closest to the people and most abundant in our country, are hospitals/clinics that take care of their clients' most basic health concerns. They are managed by the Local Governments Areas and offer no specialist care. The secondary health facilities (SHFs) are state government-controlled facilities. They are often better equipped/staffed than the PHFs and offer some degree of specialist care. The tertiary health facilities (THFs) are federal/state government and privately owned teaching hospitals and Federal medical centers. They are the flagship HFs in our country, offering wide range of specialist care in most cases and subspecialization services in some.

The BTF (2007 version) guidelines for the use of mannitol in $\mathrm{HI}$ were used to assess the appropriateness of its usage. ${ }^{5}$ Appropriate mannitol administration includes severe TBI (Glasgow coma score $\leq 8$ ), neurological deterioration under observation (GCS score drop of $\geq 2$ ), or documented/evolving anisocoria. Infusion time of $\geq 60$ minutes was defined as nonbolus mannitol administration. $\approx$ Patients with HI with the following characteristics were not included in this study: those who presented directly to our hospital, those without a referral letter, and those who were not given mannitol or in whom their pre-neurosurgical care mannitol administration status was unknown. The commercial statistical software, the Statistical Package for the Social Sciences, version 22 (SPSS Science Inc., Chicago, Illinois, United States), was used for the data analysis. An $\alpha$-value of $<0.05$ was deemed significant for the inferential statistical tests.

\section{Results}

Seventy-one patients, 53 males and 18 females (M:F $\approx 3: 1$ ) were recruited (-Table 1 ). More than half of the patients (54.9\%) were between 20 and 49 years of age ( mean $=36.8$ years). Seventeen patients $(23.9 \%)$ were referred from privately-owned hospitals, 13 (18.3\%) from PHFs, 20 (28.2\%) from SHFs, and 21 (29.6\%) from THFs (-Table 1). HI was mild (GCS 13-15) in 13 patients (18.3\%), moderate (GCS 9-12) in 29 (40.8\%), and severe (GCS $\leq 8$ ) in 29 (40.8\%) patients. Pupillary abnormalities were documented in five patients (7.04\%), all severely head injured. Neurological 
deterioration was documented in two patients, both with mild HI.

Based on our criteria in "Methods" indication for mannitol administration was deemed appropriate in less than half, $31 / 71$ (43.7\%), of the patients. Of these cases with appropriate indications for mannitol administration, 29/31 (93.6\%) had severe $\mathrm{HI}$ and $2 / 31$ (6.4\%) were those with mild HI who later suffered neurological deterioration (-Table 1). With respect to the status of the referring HF, appropriate indication for mannitol administration was seen in $8 / 17$ (47.1\%) of patients referred from private hospitals, $3 / 13$ (23.1\%) from PHF, 9/20 (45.0\%) from SHF, and 11/21 (52.4\%) from THF (-Fig. 2).

Data on mannitol dosing was available in 60.6\% (43/71) of the patients. Out of this population, $8 / 43$ patients $(18.6 \%)$ were given $10 \%$ mannitol as a continuous infusion: four (50\%) referred from PHF and two patients (25\%) apiece from private hospitals and SHF. Mannitol was administered

Table 1 Mannitol dosing error during pre-neurosurgical care of the head injured: clinical and demographic characteristics of the patients

\begin{tabular}{|c|c|}
\hline Variables & Frequency (\%) \\
\hline \multicolumn{2}{|l|}{ Gender $(N=71)$} \\
\hline Male & $53(74.6)$ \\
\hline Female & $18(25.4)$ \\
\hline \multicolumn{2}{|l|}{ Severity of injury $(N=71)$} \\
\hline Mild & $13(18.3)$ \\
\hline Moderate & $29(40.8)$ \\
\hline Severe & $29(40.8)$ \\
\hline \multicolumn{2}{|l|}{ Source of referral $(N=71)$} \\
\hline Private hospital & $17(23.9)$ \\
\hline Primary health facility & $13(18.3)$ \\
\hline Secondary health facility & $20(28.2)$ \\
\hline Tertiary health facility & $21(29.6)$ \\
\hline \multicolumn{2}{|l|}{ Indication for mannitol } \\
\hline \multicolumn{2}{|l|}{ Administration $(N=71)$} \\
\hline Appropriate & $31(43.7)$ \\
\hline Inappropriate & $40(56.3)$ \\
\hline \multicolumn{2}{|l|}{ Percentage of mannitol } \\
\hline \multicolumn{2}{|l|}{ Administered $(N=43 / 71)$} \\
\hline $10 \%$ & $8(18.6)$ \\
\hline $20 \%$ & $35(81.4)$ \\
\hline \multicolumn{2}{|l|}{ Dosing of mannitol $(N=43 / 71)$} \\
\hline Appropriate & $13(30.2)$ \\
\hline Inappropriate & $30(69.8)$ \\
\hline \multicolumn{2}{|l|}{ Dosing error $(N=30 / 43)$} \\
\hline Nonbolus administration & $23(76.7)$ \\
\hline Overdosing & $7(23.3)$ \\
\hline Underdosing & 0.0 \\
\hline
\end{tabular}

as a $20 \%$ solution in the rest, that is, $35 / 43$. Of these, dosing error occurred in $62.9 \%$ (22/35). This error was overdosing in $7 / 35$ (20\%), and nonbolus administration in 15/35 (42.9\%). Analyzing this dosing error with respect to the referring HF revealed that the error was more likely to occur in the private hospitals $(p<0.01)$ : all the $13(100 \%)$ patients from private hospitals, 6/9 (66.7\%) from PHF, 6/10 (60\%) from SHF, and 5/11 (45.5\%) of the patients from THF (-Fig. 3; - Table 2). There was no relationship between the age of the patients and appropriateness of mannitol administration ( $p=0.841$; - Table 3), or dosing error ( $p=0.354$; - Table 4). All but one of the patients were given mannitol prior to brain imaging. Urethral catheterization pre- or after administration of the mannitol was done in 40 patients (56.3\%) only.

\section{Discussion}

One of the cardinal aims of treatment of brain trauma is prevention of secondary assault of which raised ICP is an important cause. Mannitol is probably the most widely administered osmotic diuretic to control raised ICP in clinical/surgical neurological practice, especially for brain trauma patients. ${ }^{5}$ There is dearth of evidence concerning the clinical administration of mannitol in the initial care of $\mathrm{HI}$ in

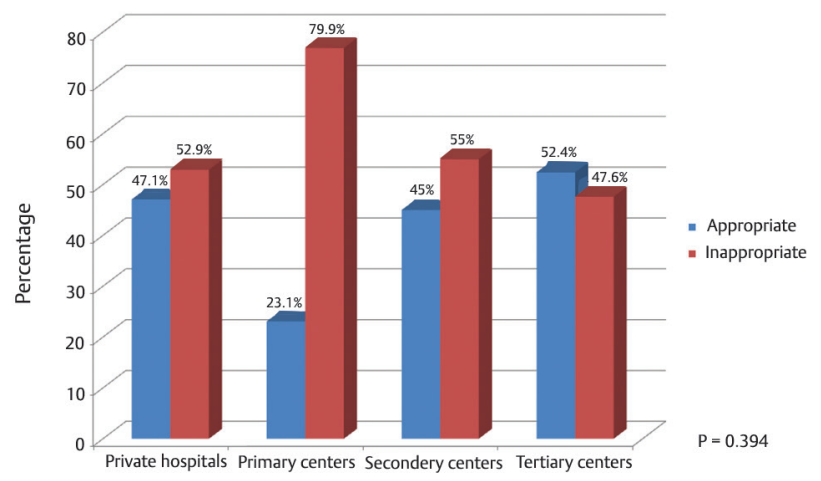

Fig. 2 Mannitol dosing error during pre-neurosurgical care of the head injured: appropriateness of the mannitol administration by respective health facilities.

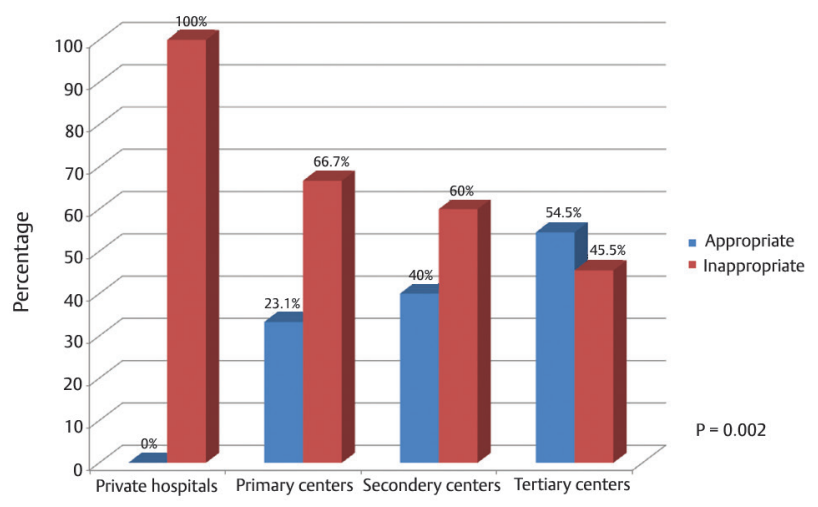

Fig. 3 Mannitol dosing error during pre-neurosurgical care of the head injured: appropriateness of the administered dose by respective health facilities. 
Table 2 Mannitol dosing error during pre-neurosurgical care of the head injured: relationship between referring hospital and dosing error

\begin{tabular}{|l|l|l|l|}
\hline \multirow{2}{*}{ Referring hospital } & \multicolumn{2}{|c|}{ Dosing error $(N=43 / 71)$} & \multirow{2}{*}{ Fisher's exact test $p$-Value } \\
\cline { 2 - 3 } & Yes & No & \\
\hline Private & $13(100)$ & $0(0.0)$ & 0.002 \\
\hline Primary & $6(66.7)$ & $3(33.3)$ & \\
\hline Secondary & $6(60.0)$ & $4(40.0)$ & \\
\hline Tertiary & $5(45.5)$ & $6(54.5)$ & \\
\hline
\end{tabular}

Table 3 Mannitol dosing error during pre-neurosurgical care of the head injured: relationship between age of the patients and appropriateness of mannitol administration

\begin{tabular}{|l|l|l|l|l|}
\hline \multirow{2}{*}{ Age (years) } & \multicolumn{2}{|c|}{ Appropriateness of mannitol administration $(N=71)$} & \multirow{2}{*}{ Chi-Square } & \multirow{2}{*}{$p$-Value } \\
\cline { 2 - 3 } & Yes & No & & \\
\hline $0-18$ & $6(46.2)$ & $7(53.8)$ & 0.040 & 0.841 \\
\hline$>18$ & $25(43.1)$ & $33(56.9)$ & & \\
\hline
\end{tabular}

Table 4 Mannitol dosing error during pre-neurosurgical care of the head injured: relationship between age of the patients and dosing error

\begin{tabular}{|l|l|l|l|l|}
\hline \multirow{2}{*}{ Age (years) } & \multicolumn{2}{|c|}{ Dosing error $(N=43 / 71)$} & \multirow{2}{*}{ Chi-square } & \\
\cline { 2 - 5 } & Yes & No & & 0.354 \\
\hline $0-18$ & $5(45.5)$ & $6(54.5)$ & 0.860 & \\
\hline$>18$ & $15(46.9)$ & $17(53.1)$ & & \\
\hline
\end{tabular}

non-neurosurgical HFs, and its use in this setting may even be counterproductive. ${ }^{5}$ The 2016 BTF guidelines suggest that mannitol use in the absence of invasive monitoring of the ICP should be addressed to clinical situations suggestive of impending intracranial brain herniations and should be given at a dose of 0.25 to $1 \mathrm{~g} / \mathrm{kg}$ body weight. ${ }^{10}$ We have shown in this study the apparent existence of a significant burden of the medical error of inappropriate use and dosing of mannitol in by the first-care responders in the management of the head injured referred to our neurosurgical practice.

The male gender-predilection as well as the higher occurrence of the young and economically mobile patients in this study subjects is in agreement with various previous reports on $\mathrm{HI}$ in the developing countries. ${ }^{2,12,13}$ The relative paucity of mild head injured patients in this study is, however, at variance with the widely reported predominance of this class of $\mathrm{HI}$ in many studies. ${ }^{1}$ But this finding agrees with an earlier series on a similar neurotrauma patient-cohort managed by us and may reflect the category of head injured patients that the nonspecialist first-responders would find themselves incapable of giving the required neurosurgical care. ${ }^{3}$ The high proportion of referrals from other THFs in this study (29.6\%) is similar to the $28.3 \%$ in the series mentioned earlier and may be due to the inadequacies of the neurosurgical infrastructure (personnel and logistics) in many of them. ${ }^{3}$

We found appropriate indication for mannitol therapy in their initial care in less than half $(43.7 \%)$ of our study subjects. Majority of these patients (93.6\%) had severe HI, while two patients (6.4\%) were the mildly head injured who deteriorated neurologically. The high rate of inappropriate administration of mannitol in our study is at variance with the study by Elliott et al who found a clear indication for mannitol in close to three-quarters (72\%) of their patients. ${ }^{6}$ But similar to our findings, severe $\mathrm{HI}$ was the most common indication for the inappropriate mannitol use in their study. We found no analytical difference in the high rates of unjustifiable mannitol use for the head injured patients among all categories of hospitals in our study, though there was a trend toward worse performance by PHF ( - Table 5).

When given appropriately as boluses, mannitol exerts a dose-dependent effect on the ICP, hence larger doses $(\geq 1 \mathrm{~g} / \mathrm{kg})$ produce greater clinical response. ${ }^{14,15}$ In contrast, infusing mannitol continuously does not effect a rapid reduction of ICP, while overdosing may result in hypovolemia and cerebral hypoperfusion thereby defeating the purpose of its administration in the pre-neurosurgical care of acute brain injury. ${ }^{6,16}$ Dosing error occurred in approximately $70 \%$ of our patients, continuous infusion accounting for about three-quarter of this figure. Our figures in this regard are significantly higher than the $22 \%$ overall dosing error, and the $7 \%$ nonbolus administration rate reported by Elliott et $\mathrm{al}^{6}$ (- Table 6). Contrary to their report, we found no case of underdosing in our own study, but a relatively higher percentage of overdosing (16.3\%) than the $7.5 \%$ they reported. Although the government hospitals did not fare satisfactorily better, dosing error was more prevalent in the private hospitals in our study. This is particularly significant because these facilities are major players in health care delivery in our country. ${ }^{17}$ 
Table 5 Mannitol dosing error during pre-neurosurgical care of the head injured: relationship between referring hospital and appropriateness of mannitol administration

\begin{tabular}{|l|l|l|l|}
\hline \multirow{2}{*}{ Referring hospital } & \multicolumn{2}{|c|}{ Appropriateness of mannitol administration $(\boldsymbol{N}=\mathbf{7 1})$} & \multirow{2}{*}{ Fisher's exact test $\boldsymbol{p}$-Value } \\
\cline { 2 - 3 } & Yes & No & \\
\hline Private & $8(47.1)$ & $9(52.9)$ & 0.785 \\
\hline Primary & $3(23.1)$ & $10(76.9)$ & 0.128 \\
\hline Secondary & $9(45.0)$ & $11(55.0)$ & 1.000 \\
\hline Tertiary & $11(52.4)$ & $10(47.6)$ & 0.433 \\
\hline
\end{tabular}

Table 6 Mannitol dosing error during pre-neurosurgical care of the head injured: comparison of our findings and the findings of Elliott et $\mathrm{al}^{6}$

\begin{tabular}{|c|l|l|}
\hline Variables & $\begin{array}{l}\text { Our study } \\
(\%)\end{array}$ & Elliott et al (\%) \\
\hline Appropriate indication & 43.7 & 72 \\
\hline Severe HI & 93.6 & 69 \\
\hline $\begin{array}{l}\text { Neurological } \\
\text { deterioration }\end{array}$ & 6.4 & 11 \\
\hline Dosing error rate & 69.8 & 22 \\
\hline Dosing error type & 76.7 & 6.7 \\
\hline $\begin{array}{l}\text { Nonbolus } \\
\text { administration }\end{array}$ & 23.3 & 7.5 \\
\hline Overdosing & 0.0 & 8.3 \\
\hline Underdosing &
\end{tabular}

Abbreviation: $\mathrm{HI}$, head injury.

Another finding of interest in this study is the administration of mannitol in all patients bar one before acquisition of brain imaging. This finding, in the light of the high rate of unjustifiable use, suggests a prevalent routine use of mannitol that is not evidence-driven in any way, in the management of $\mathrm{HI}$ in our environment. Mannitol was administered without urethral catheterization to monitor urinary output either, despite the diuretic property and its potential to cause hypovolemia and renal impairment. In a particular case the patient was given mannitol despite documented hypotension at presentation.

Overall, our study on the use of mannitol in the initial treatment of patients with $\mathrm{HI}$ by the first-responders in our country, an LMIC, showed data indicating apparent abuse of this all-important drug agent by these non-neurosurgeons. The apparent better practice, and that only marginally so, in the cohort referred from THFs may be due to the more academic nature of these facilities.

This study, nevertheless, is limited in some ways, including the small sample size, and especially our reliance on the documentations in the referral notes, which were inadequately written in some cases, for interrogating the mannitol stewardship by the referring hospitals. Indeed, the problem might actually be under-reported in this study from the point of view of cases in which the details of the pre-neurosurgical care were not available among the study subjects.

\section{Conclusion}

In a significant number of cases, the use of mannitol in the pre-neurosurgical care of the head injured appears to follow no specific indication and is fraught with dosing errors in our environment. This, as to be expected, potentially exposes the patients to untoward effects and needlessly increases the costs of care. This practice seems to be all pervasive across all the strata of our national health care services, primary to tertiary. There is thus a need for continuing medical education of these cadres of health care givers on this subject in our environment.

\section{Authors' Contributions}

A.O. A.: concept, design, definition of intellectual content, literature search, data acquisition, data analysis, statistical analysis, manuscript preparation, manuscript editing, manuscript review, and guarantor; T. A. O.: concept, design, definition of intellectual content, literature search, data acquisition, data analysis, statistical analysis, manuscript preparation, manuscript editing, and manuscript review; T. A. M.: concept, definition of intellectual content, literature search, data acquisition, data analysis, statistical analysis, manuscript preparation, manuscript editing, and manuscript review; and O. E. O.: concept, definition of intellectual content, data acquisition, data analysis, statistical analysis, manuscript preparation, manuscript editing, and manuscript review.

\section{Funding}

None.

\section{Conflict of Interest}

None declared.

\section{References}

1 Adeleye AO, Olowookere KG, Olayemi OO. Clinicoepidemiological profiles and outcomes during first hospital admission of head injury patients in Ikeja, Nigeria. A prospective cohort study. Neuroepidemiology 2009;32(2):136-141

2 Li M,Zhao Z, Yu G, ZhangJ. Epidemiology of traumatic brain injury over the world: a systematic review. Gen Med 2016;4:e275-e275

3 Oyemolade TA, Malomo TA, Adeleye AO. Indiscriminate use of antibiotics in neurotrauma patients referred for expert neurosurgical care in a developing country. J Neurosci Rural Pract 2019;10(4):653-656 
4 Adeleye AO, Okonkwo DO. Inter-hospital transfer for neurosurgical management of mild head injury in a developing country: a needless use of scarce resources? The Indian J. Neurotrauma. 2011;8:1-5

5 Wakai A, McCabe A, Roberts I, Schierhout G. Mannitol for acute traumatic brain injury. Cochrane Database Syst Rev 2013;(8):CD001049

6 Elliott CA, MacKenzie M, O’Kelly CJ. Mannitol dosing error during interfacility transfer for intracranial emergencies. J Neurosurg 2015;123(5):1166-1169

7 Shawkat H, Westwood MM, Mortimer A. Mannitol: a review of its clinical uses. Contin Educ Anaesth Crit Care Pain 2012;12:82-85

8 Cheng $\mathrm{F}$, Xu M, Liu $\mathrm{H}$, Wang $\mathrm{W}$, Wang $\mathrm{Z}$. A retrospective study of intracranial pressure in head-injured patients undergoing decompressive craniectomy: a comparison of hypertonic saline and mannitol. Front Neurol 2018;9:631

9 Fandino W. Understanding the physiological changes induced by mannitol: from the theory to the clinical practice in neuroanaesthesia. J Neuroanaesth Crit Care 2017;4(3):138-146

10 Carney N, Totten AM, O'Reilly C, et al. Guidelines for the management of severe traumatic brain injury, fourth edition. Neurosurgery 2017;80(1):6-15
11 Adeleye AO. Pattern of referrals of head injury to the University College Hospital, Ibadan. Ann Ib Postgrad Med 2017; 15(1):34-40

12 Adeleye AO, Ogun MI. Clinical epidemiology of head injury from road-traffic trauma in a developing country in the current era. Front Neurol 2017;8:695

13 Adeleye AO, Clark DJ, Malomo TA. Trauma demography and clinical epidemiology of motorcycle crash-related head injury in a neurosurgery practice in an African developing country. Traffic Inj Prev 2019;20(2):211-215

14 Fink ME. Osmotherapy for intracranial hypertension: mannitol versus hypertonic saline. Continuum (Minneap Minn) 2012;18(3):640-654

15 Sorani MD, Manley GT. Dose-response relationship of mannitol and intracranial pressure: a metaanalysis. J Neurosurg 2008;108(1):80-87

16 Cruz J, Minoja G, Okuchi K, Facco E. Successful use of the new high-dose mannitol treatment in patients with Glasgow Coma Scale scores of 3 and bilateral abnormal pupillary widening: a randomized trial. J Neurosurg 2004;100(3):376-383

17 Adeleye AO, Jite IE, Smith OA. A tale of two acute extradural hematomas. Surg Neurol Int 2016;7:54 